\title{
Epigenetically Enhanced PDT (ePDT) Induces Significantly Higher Levels of Multiple Extrinsic Pathway Apoptotic Factors than Standard PDT, Resulting in Greater Extrinsic and Overall Apoptosis of CTCL
}

\author{
Katrin A. Salva ${ }^{1}$, Youn H. Kim² ${ }^{2}$ Ziba Rahbar $^{2}$, and Gary S. Wood ${ }^{\star}, 1,3$ \\ ${ }^{1}$ University of Wisconsin, Department of Dermatology, Madison WI \\ ${ }^{2}$ Stanford University, Department of Dermatology, Stanford CA \\ ${ }^{3}$ VA Medical Center, Madison WI
}

\section{Abstract}

\begin{abstract}
Aminolevulinate-based photodynamic therapy (ALA-PDT) selectively eliminates diseased tissues primarily through the induction of intrinsic apoptotic pathway. ALA-PDT is a first-line therapy for actinic keratosis, however, it is less effective for cutaneous T-cell lymphoma (CTCL). We have previously demonstrated that the resistance of CTCL to apoptosis correlates with decreased expression of death receptors such as FAS, and that methotrexate functions as an epigenetic regulator that re-establishes the susceptibility of CTCL to extrinsic pathway apoptosis. We showed previously that MTX augments the effectiveness of PDT by sensitizing cells to apoptosis by induction of apoptotic factors, a process we call "epigenetically enhanced" PDT (ePDT). Here, in CTCL cell lines, leukemic CTCL cells and normal blood T cells, we analyzed multiple components of the FAS, TRAIL and TNF families using multispectral imaging of immunostained cytopreparations, a quantitative technique with 5 -fold greater sensitivity than standard immunocytology. ePDT induced significantly greater FAS, FASL, TRAIL-R1 \& -R2, and TNFa levels than standard PDT. This correlated with significantly greater induction of extrinsic pathway apoptosis and/or overall apoptosis in all CTCL samples. There was no appreciable effect on normal $\mathrm{T}$ cells. These data set the stage for clinical trials of $\mathrm{ePDT}$ as a novel localized treatment of CTCL.
\end{abstract}

\section{Graphical abstract}

Aminolevulinate photodynamic therapy induces intrinsic apoptosis in targeted tissues and eradicates actinic keratoses but is less effective for cutaneous T-cell lymphoma (CTCL). We have previously shown that a) the apoptotic resistance of CTCL correlates with decreased death receptor expression, b) methotrexate (MTX) epigenetically enhances extrinsic apoptosis, and c) MTX augments PDT by upregulating apoptotic factors, a process called "epigenetically enhanced" PDT (ePDT). Here, ePDT induced significantly greater FAS, FASL, TRAIL-R1 \& -R2, and TNFa

"Corresponding author's gwood@ dermatology.wisc.edu (Gary S. Wood). SUPPORTING INFORMATION

Additional Supporting Information is available in the online version of this manuscript: 
levels as well as extrinsic and/or overall apoptosis than standard PDT in CTCL cell lines/leukemic cells. These data support clinical trials of ePDT for CTCL.

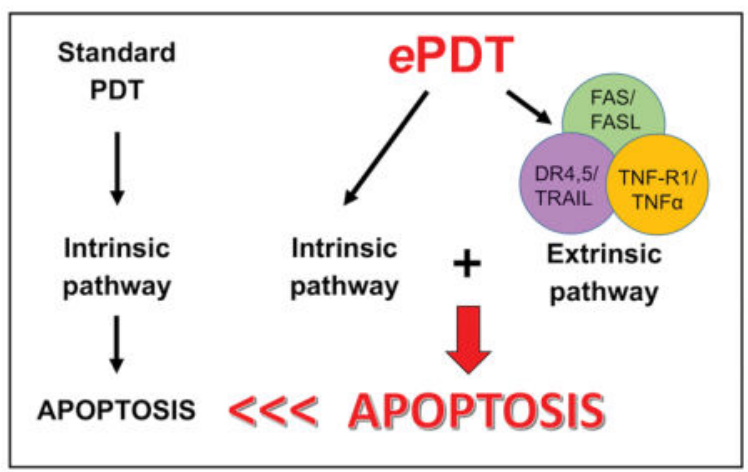

\section{INTRODUCTION}

In photodynamic therapy (PDT), the build-up of a photosensitizing agent in diseased cells followed by exposure to visible light results in intracellular generation of reactive oxygen species (ROS) (1-4). ROS induce primarily intrinsic (mitochondrial), caspase 9-mediated apoptosis (1-3). In topically applied aminolevulinate (ALA)-PDT, the porphyrin precursor ALA is metabolized by the targeted tissue into protoporphyrin IX (PpIX), an endogenous photosensitizer (3). The selectivity and the non-invasive nature of topical ALA-PDT as well as the absence of serious and/or long-term side effects render this modality one of the most attractive treatment options for malignant and benign chronic skin disorders $(3,4)$. However, while actinic keratoses and superficial basal cell carcinomas respond exceedingly well to ALA-PDT, comparable results have not yet been achieved in diseases in which neoplastic and/or inflammatory $\mathrm{T}$ cells play a fundamental role, such as chronic psoriasis, lichen planus or cutaneous T cell lymphoma (CTCL) (4-8).

In CTCL, the resistance to apoptosis is inversely correlated with decreased expression of several apoptotic factors such as the death receptor FAS (9-11). We have previously discovered the function of MTX as an epigenetic regulator that re-establishes the susceptibility of treatment-resistant malignant $\mathrm{T}$ cells to extrinsic apoptosis (11-13). Using pyrosequencing, we showed that MTX reduces methylation of the promoter region of apoptosis genes such as FAS. We also have an abstract in press in the Journal of Investigative Dermatology that shows MTX as well as knockdown of DNA methyltransferases 1 and $3 \mathrm{~A}$ increase expression of FASL.

Recently, we demonstrated that the combination of MTX with conventional PDT in the form of epigenetically enhanced PDT (ePDT) activates both the intrinsic as well as the extrinsic apoptotic pathway, thereby inducing significantly greater cell death in CTCL as opposed to MTX or ALA-PDT alone (14). Here, we extend our previous studies by analyzing multiple components of the FAS, TRAIL and TNF families after PDT alone versus ePDT in CTCL cell lines, leukemic CTCL cells and normal blood T cells with multispectral imaging analysis (MIA), a quantitative technique that is superior to conventional immunohistochemistry for the detection of antigens (15). 


\section{MATERIALS AND METHODS}

\section{Human CTCL cell lines}

The CTCL cell lines derived from patients with mycosis fungoides (MyLa, HH) or Sézary syndrome (Hut78, SZ4 and SeAx) were acquired as outlined elsewhere $(11,13)$. These cell lines are characterized by known anomalies of the FAS gene, including its absence (SeAx cells), and therefore show varying levels of FAS expression (11-13). For cell culture, RPMI 1640 media with $2 \mathrm{mM} \mathrm{L}$-glutamine with $10 \%$ fetal bovine serum and $1 \mathrm{mM}$ sodium pyruvate (added to all cell lines) and 10mM 4-(2-hydroxyethyl)-1-piperazine-ethanesulfonic acid (HH and SZ4) was used.

\section{Human leukemic CTCL cells}

Cryopreserved peripheral blood mononuclear cells were obtained from a woman with Sézary syndrome stage IVA. The patient gave written informed consent and all protocols adhered to the Declaration of Helsinki principles. At the time of blood collection, the patient was 3 months off therapy. Dominant clonality was confirmed by both TCR-beta and TCRgamma gene rearrangement analysis. The sample consisted of $97 \%$ tumor cells as assessed by flow cytometric detection of the $\mathrm{CD} 3+\mathrm{CD} 4+\mathrm{CD} 26-$ phenotype. After thawing, the cells were suspended in warm RPMI 1640 medium with $2 \mathrm{mM}$ L-glutamine and $10 \%$ FBS, pelleted by centrifugation, re-suspended in medium and cultured as described above.

\section{Human normal blood T cells}

Cryopreserved, negatively selected human CD4+ T cells from three normal, healthy US donors (Precision Bioservices, Frederick, MD) were thawed and cultured in accordance with the protocol provided by the manufacturer. Standard ALA-PDT and ePDT: Standard PDT was performed by incubating the cells for 6 hours with $1 \mathrm{mM} 5$-aminolevulinate hydrochloride (Sigma-Aldrich, Saint Louis, MO) with subsequent illumination with $630 \mathrm{~nm}$ light at $3.22 \mathrm{~J} / \mathrm{cm}^{2}$ via the Luzchem Expo Panel light source, which was outfitted with a red filter (Luzchem Research, Ottawa, Canada). Cells were collected at 24 hours after light exposure. For ePDT, cells were first exposed to $100 \mathrm{nM}$ methotrexate hydrate (SigmaAldrich) for 48 hours and then treated with ALA-PDT as outlined above. All experiments included untreated controls, ALA only, light only as well as MTX only samples. To prevent photobleaching, all steps requiring ALA were carried out in the dark. To monitor for cellular PpIX, cells were collected after 6 hours of incubation with ALA, centrifuged, re-suspended in phosphate-buffered saline (PBS) and transferred onto glass slides to visualize and measure the PpIX fluorescence with the Nuance microscope. Using a 405nm light source and the fluorescence mode, multispectral images cubes were generated at $20 \mathrm{X}$ magnification. The spectral library was created by sampling the characteristic fluorescence of PpIX. Levels were assessed as average signal/cell.

\section{Immunohistochemistry (IHC)}

After collection and washing with PBS, the cells were centrifuged and re-suspended in PBS to produce cytopreparations on positively charged microscope glass slides. The following panel of primary antibodies was used for biomarker detection: anti-FAS/CD95 antibody 
clone APO-1-1 (Enzo Life Sciences, Farmingdale, NY), anti-FASL antibody clone 5G51 (Enzo Life Sciences), anti-TRAIL antibody clone III6F (Enzo Life Sciences), anti-DR4 antibody clone DJR1 (Thermo Fisher Scientific, Waltham, MA), anti-DR5 polyclonal antibody (Novus Biologicals, Littleton, CO), anti-TNF-R1 antibody clone H398 (Enzo Life Sciences), anti-TNFa antibody clone 2C8 (Abcam, Cambridge, MA), anti-cleaved caspase-3 antibody clone 31A1067 (Enzo Life Sciences), anti-cleaved caspase-8 antibody clone 12F5 (Enzo Life Sciences), anti-cleaved caspase-9 polyclonal antibody (Abcam). Methylene blue (MB) was applied to all slides as counterstain. Protein visualization was performed with the MACH4 Universal HRP-Polymer detection assay (Biocare Medical, Concord, CA) and 3,3diaminobenzidine (DAB).

\section{Multispectral imaging analysis (MIA)}

Protein expression was quantitated with Nuance (Perkin-Elmer, Waltham, MA). A detailed description is outlined in our previous article (14). Briefly, following the acquisition of multispectral image cubes of relevant slide areas, a spectral library with wavelength curves of individual dyes was generated using slides stained with $\mathrm{MB}$ alone and $\mathrm{DAB}$ alone. Blue and red pseudo-colors were chosen to represent MB and DAB, respectively. 100-300 cells/ slide were marked for analysis. Nuance determined the amount of target protein (the intensity of red color) within each cell using the spectral library as reference, showing final measurements as average optical density (OD)/cell. All Tables and Figures are based on quantitation of protein expression by MIA.

\section{Statistical analysis}

Statistical analysis was performed by using one-way ANOVA followed by Tukey's multiple comparison test. P-values $<0.05$ were considered statistically significant.

\section{RESULTS \\ ePDT induces significantly higher expression of death receptors/ligands in vitro and ex vivo than standard PDT}

As summarized in Table 1, the levels of various death receptors/ligands in CTCL cell lines and leukemic CTCL cells were significantly higher after ePDT versus PDT alone.

Supporting Information Figure S1 depicts representative microscopic IHC images and highpower images of biomarker expression generated by Nuance as well as examples of quantitative analysis.

FAS and FASL (Figure 1)-In FAS-high MyLa and Hut78 cells, the expression of this receptor remained unaffected by all therapies (i.e. MTX alone, PDT alone, ePDT). In the FAS-low cell lines HH and SZ4, MTX alone as well as ePDT, but not standard PDT, led to a significant upregulation of FAS. The SeAx cells (FAS-null) were not included in this set of experiments. In the PBMCs from the patient with Sézary syndrome, baseline FAS expression was low and did not change in response to all treatment modalities tested. The CTCL cell lines were characterized by low baseline levels of FASL. In contrast, untreated leukemic CTCL cells were FASL-high. PDT alone as well as ePDT resulted in an increased FASL expression in all five CTCL cell lines, with ePDT inducing significantly higher FASL 
levels relative to standard PDT in MyLa, Hut78 and SZ4, while neither treatment altered FASL expression in leukemic PBMCs.

DR4, DR5 and TRAIL (Figure 2)—Low baseline expression of DR4 and its significant induction in response to MTX alone were observed in all cell types. PDT alone led to increased DR4 levels in MyLa, SZ4 and SeAx, but not in Hut78, HH and patient-obtained cells. In all samples, ePDT led to significantly higher DR4 induction as opposed to standard PDT. Four CTCL cell lines (Hut78, HH, SZ4 and SeAx) exhibited high baseline DR5 expression, which did not change post-treatment. DR5 expression was significantly induced in MyLa following MTX exposure as well as post ePDT in MyLa and in leukemic CTCL cells. Among untreated samples, SZ4 showed high levels of TRAIL, the binding partner of DR4 and DR5. In MyLa, significantly greater TRAIL expression was measured after MTX alone as well as after ePDT, but not after PDT alone. In HH and in leukemic PBMCs, TRAIL was elevated in response to both standard PDT and ePDT relative to controls, however, there was no significant difference in TRAIL induction between the two modalities.

TNF-R1 and TNFa (Figure 3)-The baseline levels of TNFR1 were low in all untreated samples. The expression of this death receptor was greatly increased following PDT alone and ePDT compared with controls, but our data showed no significant difference in TNFR1 levels between ALA-PDT- versus ePDT-treated cells. Baseline TNFa expression was low in all cells and was markedly elevated in all MTX only- as well as all ePDT-treated samples. In MyLa, Hut78, SZ4 and SeAx cell lines, TNFa was also upregulated in response to conventional PDT. As shown in Table 1, ePDT resulted in significantly higher TNFa levels than ALA-PDT in all cells except MyLa.

\section{ePDT results in greater extrinsic pathway apoptosis than standard PDT in CTCL cell lines as well as in leukemic CTCL cells}

ePDT produced significantly greater caspase 8 activation in CTCL cell lines and in patientderived leukemic PBMCs as opposed to standard PDT as well as to MTX alone (Figure 4). These data indicate that $e \mathrm{PDT}$ induces primarily the extrinsic apoptotic pathway in malignant $\mathrm{T}$ cells both in vitro and ex vivo.

\section{ePDT results in greater caspase 3 cleavage than standard PDT in CTCL cell lines, and induces greater caspase 9 cleavage in Hut78 and HH cells, thereby enhancing the apoptotic efficacy of this modality versus standard PDT}

Relative to conventional PDT, ePDT resulted in significantly greater overall apoptosis in all cell lines as indicated by caspase 3 cleavage following treatment (Figure 5). The greatest increase in apoptotic cell death after ePDT as opposed to standard PDT was measured in $\mathrm{HH}$ and SZ4, the FAS-low CTCL prototypes. Notably, in the ex vivo setting there was also more overall apoptosis post $e \mathrm{PDT}$ as opposed to PDT alone that was just short of statistical significance $(p=0.067)$. Moreover, ePDT resulted in greater induction of intrinsic apoptosis, evidenced by caspase 9 activation, in two CTCL cell lines, namely Hut78 and HH (Figure 6). Table 2 summarizes the significant differences in the expression of apoptotic markers (i.e. cleaved caspase 3-, 8- and 9-products) after ePDT relative to standard PDT. 


\section{ePDT does not significantly affect the expression of death receptor/death receptor ligands in normal T cells, nor does it result in a significant increase of cleaved caspase-3, -8 or -9 products in normal blood $\mathrm{T}$ cells}

The baseline expression of death receptor/death receptor ligands in normal blood $\mathrm{T}$ cells remained unchanged after exposure to MTX, ALA-PDT and ePDT. Furthermore, neither treatment caused significant apoptosis as assessed by cleavage of caspases in these lymphocytes (Figure S2).

\section{DISCUSSION}

Curative therapies for MF and its leukemic form, Sézary syndrome (SS), which comprise the vast majority of all CTCLs, are currently lacking. Moreover, the indolent clinical course of early-stage MF requires effective yet minimally toxic therapeutic strategies. Thus far, the application of standard ALA-based PDT in CTCL has resulted in variable clinical outcomes despite adequate application of photosensitizer and light $(4-8,16)$. The sensitivity of malignant $\mathrm{T}$ cells to PDT-induced apoptotic cell death is well documented (17-22). Nevertheless, the underlying biomolecular mechanisms remain to be investigated in detail. ALA-PDT activates primarily the intrinsic, caspase 9-mediated apoptotic pathway, however, changes in the expression of death receptors/ligands and/or caspase 8-activation have been described in oral and lung cancer cells as well as in the setting of PDT with drugs other than ALA, respectively (23-28). Furthermore, most studies investigating the response of malignant T-lymphocytes to PDT involve the Jurkat cell line, which originates from T-cell acute lymphoblastic leukemia and therefore does not represent CTCL concerning its tumor biology $(17,29)$. Our experiments, however, were carried out using human CTCL cell lines and ex vivo leukemic CTCL cells characterized by a wide range of death receptor/ligand expression, thereby creating ideal conditions to analyze the associations between varying levels of the above-mentioned markers and the effectiveness of MTX, PDT and ePDT in inducing apoptosis related cytotoxicity in CTCL. Our current report includes data on upstream extrinsic (caspase 8) and intrinsic (caspase 9) apoptotic pathways. It also includes data on the downstream apoptotic pathway (caspase 3) into which both of these upstream pathways flow. Activation of both upstream and downstream apoptotic factors by their cleavage is considered reliable evidence of cytotoxicity via apoptosis. Furthermore, our earlier ePDT paper demonstrated increased PARP cleavage products accompany the cleavage of these apoptotic factors. This is a second independent confirmation of cytotoxicity.

Epigenetic alterations involving death receptor/ligand pairs like FAS/FASL, TNF-R1/TNFa and TRAIL-R2(DR5)/TNF-related apoptosis-inducing ligand (TRAIL) represent a major mechanism of apoptotic resistance in malignant $\mathrm{T}$ cells. Moreover, variable expression of apoptotic pathway components in lesional skin of patients with MF points to heterogeneity of such alterations among individuals (30). Therefore, the relative lack of therapeutic success of PDT in CTCL patients could be caused by epigenetic suppression of death receptor-/ligand-expression. Our previously published ePDT study (14) supports the above hypothesis, demonstrating significantly greater apoptosis in 5 human CTCL cell lines post ePDT versus PDT or MTX alone. Specifically, we observed that a) the apoptotic response of 
CTCL correlated positively with baseline FAS expression and that $e$ PDT reestablished FAS expression in FAS-low cell lines while simultaneously upregulating FASL, b) ePDT also increased some other extrinsic apoptotic pathway components in different CTCL cell lines (although non-FAS pathway analysis was limited in our earlier study), and c) ePDT enhanced primarily extrinsic as opposed to intrinsic apoptosis in CTCL. Thus, ePDT eliminates the targeted cells by inducing both the extrinsic as well as the intrinsic apoptotic pathways, whereas standard PDT induces primarily intrinsic apoptosis. These studies led to the introduction of ePDT as a novel therapeutic tool for disorders in which apoptosisresistant T-lymphocytes play a major role (14).

The data presented in the current manuscript expands our previous work on ePDT that was purely an in vitro study of CTCL cell lines and examined only a few apoptosis factors. In addition to confirming this prior work, the current paper extends our findings to the wide array of death receptor/ligand pairs like FAS, TRAIL and TNF apoptotic pathways. Importantly, it also adds ex vivo data from CTCL leukemic blood cells and normal blood T cells. These are primary isolates, not cell lines. They are the closest substitutes for actual clinical data because no good animal models of human CTCL exist.

As demonstrated in Table 2, Figure 4 and Figure 5, ePDT led to greater extrinsic and overall apoptosis both in vitro and ex vivo as compared to standard PDT. This corresponded with markedly greater induction of various death receptor/death receptor ligands post $e$ PDT versus PDT alone, as summarized in Table 1. The histograms in Figures 1-3 illustrate the changes of death receptor/death receptor ligand expression observed post exposure to the single components of ePDT. Standard PDT did not affect the expression of FAS in CTCL. However, ePDT upregulated FAS in FAS-low HH and SZ4 cells. While PDT alone resulted in increased levels of FASL in all 5 CTCL cell lines, ePDT induced significantly more in MyLa, Hut78 and SZ4. In contrast, FAS/FASL expression remained unchanged from relatively high baseline levels in the leukemic CTCL cells, suggesting the involvement of additional death receptor/ligand pairs in the enhanced response to ePDT ex vivo in this particular CTCL patient. Relative to standard PDT, ePDT led to significantly increased expression of DR4 (all five CTCL cell lines, leukemic CTCL cells), DR5 (MyLa, leukemic CTCL cells) and TRAIL (MyLa). Furthermore, in all CTCL samples except MyLa, ePDT induced greater TNFa levels than PDT. Collectively, these results show that the combination of MTX and traditional PDT in the form of ePDT results in greater induction of various death receptor/ligand pairs. Moreover, while all malignant $\mathrm{T}$ cells demonstrated increased cleavage of caspase 9 in response to MTX, PDT and ePDT, intrinsic apoptosis was significantly greater post ePDT versus standard PDT in two of the cell lines tested (Table 2, Figure 6). This could be due to the fact that MTX, independently of its epigenetic actions, also tends to induce ROS production, and/or that caspase 8-cleavage is known to activate the intrinsic pathway, e.g. via the truncation of BID (BH3 interacting-domain death agonist) (31, 32). Since MTX is known to increase PpIX in tumor cells, increases in cleaved caspase 9 could also be due to this mechanism $(33,34)$. This is consistent with MTX's known effect on inducing intrinsic (mitochondrial) apoptosis through increased reactive oxygen species resulting in cleavage of caspase 9. However, this effect does not alter our findings showing that MTX in the context of ePDT augments apoptosis by increasing death receptor/ligand pairs resulting in increased extrinsic apoptosis. Our current data clearly demonstrate that 
ePDT increases cleaved caspase 8 (the hallmark of extrinsic apoptosis). If increasing PpIX was the main effect of MTX, then cleaved caspase 8 should not be increased because PpIX does not act through the caspase 8 pathway. Importantly, as shown in Figure S2, neither ePDT nor its single components caused significant apoptosis or measurable changes in the expression of death receptors or their respective ligands in normal blood $\mathrm{T}$ cells derived from healthy individuals. These observations suggest that the selective elimination of malignant versus normal cells, which represents an especially advantageous attribute of PDT over conventional chemotherapy, applies to ePDT as well.

The current report, along with our previous studies, sets the stage for clinical trials of ePDT. Animal studies are also an option; however, there are really no good animal models of human CTCL. In addition, both MTX and PDT are FDA-approved therapies, and both have been used separately to treat CTCL patients. Therefore, we believe that our current data provide a rationale for proceeding with early phase clinical trials. Nevertheless, it is important to emphasize that initial human trials should focus on establishing a dose-range that minimizes clinical toxicity before proceeding to trials focused on clinical efficacy. ePDT might benefit especially patients with uni-/oligolesional patch-/plaque-stage MF in whom minimally toxic treatment options are preferable. Notably, ePDT could be successful in cases that previously failed standard PDT. In this context, quantitative IHC of skin biopsies with MIA would serve as an optimal tool to pre-select individuals for whom ePDT would be most beneficial and to monitor its effects by objective assessment of relevant proteins directly in situ $(11,15,35-37)$. Thus, the high response rates of AKs to ALA-PDT might be reproduced in subjects with CTCL. In particular, individuals with follicular MF located in problematic areas, i.e. face, scalp, hands and feet, would represent ideal candidates for ePDT. The range of available therapeutic options in such cases is limited due to the complex topography of the lesions. Using standard ALA-PDT, Pileri et al reported partial and complete remissions of MF patches in difficult-to-treat sites (16). Goddard et al have demonstrated that low-dose, high dose-rate brachytherapy, i.e. the administration of radiation via flexible catheters secured within customized surface molds, can lead to prolonged remission in patients with facial and acral CTCL lesions $(38,39)$. Brachytherapy has also been used to treat other skin malignancies, such as squamous and basal cell carcinoma as well as cutaneous metastases of Merkel cell carcinoma $(40,41)$. However, although radiotherapy is effective in the treatment of CTCL, it carries the risk of significant acute/chronic toxicity and of inducing other cancers. In addition, cumulative toxicity limits the number of treatments to the same area over time. Here, ePDT would serve as a noninvasive and non-carcinogenic as well as a cost-effective alternative to brachytherapy. Lastly, it is possible that ePDT could also surpass the effectiveness of standard PDT in the management of other cutaneous cancers, such as basal cell or squamous cell carcinoma, which are frequently characterized by epigenetic dysregulations of apoptotic pathway components, and it would be especially useful in patients with cancers located in anatomically challenging areas.

\section{Supplementary Material}

Refer to Web version on PubMed Central for supplementary material. 


\section{Acknowledgments}

This study was supported in part by the UW SDRC grant P30AR066524 funded by the National Institute of Arthritis and Musculoskeletal and Skin Diseases (K. A. Salva, G. S. Wood), grant R21CA206104 funded by the National Cancer Institute, Merit Review funding from the Department of Veterans Affairs, the Spatz Foundation and the Swatek Family Fund (G. S. Wood).

\section{References}

1. Henderson BW, Dougherty TJ. How does photodynamic therapy work? Photochem. Photobiol. 1992; 55:145-157.

2. Ahmad N, Feyes DK, Agarwal R, Mukhtar H. Photodynamic therapy results in induction of WAF1/ CIP1P21 leading to cell cycle arrest and apoptosis. Proc Natl Acad Sci. 1998; 95:6977-6982. [PubMed: 9618524]

3. Kennedy JC, Pottier RH, Pross DC. Photodynamic therapy with endogenous protoporphyrin IX: basic principles and present clinical experience. J Photochem Photobiol B. 1990; 14:275-292.

4. Salva K. Photodynamic therapy: unapproved uses, dosages or indications. Clin Dermatol. 2002; 20:571-581. [PubMed: 12435528]

5. Bissonnette R, Lui H. Current status of photodynamic therapy in dermatology. Dermatol Clin. 1997; 15:507-519. [PubMed: 9189686]

6. Wan MT, Lin JY. Current evidence and applications of photodynamic therapy in dermatology. Clin Cosmet Investig Dermatol. 2014; 7:145-163.

7. Edstrom DW, MA, Hedblad MA. Long-term follow-up of photodynamic therapy for mycosis fungoides. Acta Derm Venereol. 2008; 88:288-290. [PubMed: 18480938]

8. Fernandez-Guarino M, Harto A, Perez-Garcia B, Montull C, De Las Heras E, Jaén P. Plaque-phase mycosis fungoides treated with photodynamic therapy: results from 12 patients. Actas Dermosifiliogr. 2010; 101:785-791. [PubMed: 21034709]

9. Braun FK, Fecker LF, Schwarz C, Walden P, Assaf C, Duerkop H, Sterry W, Eberle J. Blockade of death receptor-mediated pathways early in the signaling cascade coincides with distinct apoptosis resistance in cutaneous T-cell lymphoma cells. J Invest Dermatol. 2007; 127:2425-2437. [PubMed: 17495957]

10. Contassot E, Kerl K, Roques S, Shane R, Gaide O, Dupuis M, Rook AH, French LE. to FasL and tumor necrosis factor-related apoptosis-inducing ligand-mediated apoptosis in Sézary syndrome Tcells associated with impaired death receptor and FLICE-inhibitory protein expression. Blood. 2008; 111:4780-4787. [PubMed: 18314443]

11. Wu J, Nihal M, Siddiqui J, Vonderheid EC, Wood GS. Low FAS/CD95 expression by CTCL correlates with reduced sensitivity to apoptosis that can be restored by FAS upregulation. J Invest Dermatol. 2009; 129:1165-1173. [PubMed: 18923451]

12. Wu J, Siddiqui J, Nihal M, Vonderheid EC, Wood GS. Structural alterations of the FAS gene in cutaneous T-cell lymphoma (CTCL). Arch Biochem Biophys. 2011; 508:185-191. [PubMed: 21036138]

13. Wu J, Wood GS. Reduction of Fas/CD95 promoter methylation, upregulation of Fas protein, and enhancement of sensitivity to apoptosis in cutaneous T-cell lymphoma. Arch Dermatol. 2011; 147:443-450. [PubMed: 21173302]

14. Salva KA, Wood GS. Epigenetically enhanced photodynamic therapy (ePDT) is superior to conventional photodynamic therapy for inducing apoptosis in cutaneous T-cell lymphoma. Photochem. Photobiol. 2015; 91:1444-1451.

15. Salva KA, Wu J, Nihal M, Wood GS. Analysis of protein expression in situ using multi-spectral imaging is superior to conventional immunohistochemistry (IHC): a new paradigm for patient selection for targeted therapy. J Invest Dermatol. 2013; 133(S):169.

16. Pileri A, Sgubbi P, Agostinelli C, Infusino SD, Vaccari S, Patrizi A. Photodynamic therapy: An option for mycosis fungoides. Photodiagnosis Photodyn Ther. 2017; 20:107-110. [PubMed: 28890210] 
17. Gad F, Viau G, Boushira M, Bertrand R, Bissonnette R. Photodynamic therapy with 5aminolevulinic acid induces apoptosis and caspase activation in malignant T cells. J Cutan Med Surg. 2001; 5:8-13. [PubMed: 11281435]

18. Granville DJ, Jiang H, McManus BM, Hunt DWC. Fas ligand and TRAIL augment the effect of photodynamic therapy on the induction of apoptosis in Jurkat cells. Int Immunopharmacol. 2001; 1:1831-1840. [PubMed: 11562074]

19. Grebenova D, Cajthamlova H, Bartosova H, Marinov J, Klamovi H, Fuchs O, Hrkal Z. Selective destruction of leukemic cells by photo-activation of 5-aminolevulinic acid-induced protoporphyrin IX. J Photochem Photobiol. 1998; 4:74-81.

20. Lam M, Lee YJ, Deng M, Hsia AH, Morrissey KA, Yan C, Azzizudin K, Oleinick NL, McCormick TS, Cooper KD, Baron ED. Photodynamic therapy with the silicon phthalocyanine Pc4 induces apoptosis in mycosis fungoides and Sezary syndrome. Adv Hematol. 2010; :896161.doi: 10.1155/2010/896161 [PubMed: 21197103]

21. Ke MS, Xue L, Feyes DK, Azizuddin K, Baron ED, McCormick TS, Mukhtar H, Panneerselvam A, Schluchter MD, Cooper KD, Oleinick NL, Stevens SR. Apoptosis mechanisms related to the increased sensitivity of Jurkat T-cells vs A431 epidermoid cells to photodynamic therapy with the phthalocyanine Pc4. Photochem Photobiol. 2008; 84:407-414. [PubMed: 18221452]

22. Jiang H, Granville DJ, North JR, Richter AM, Hunt DWC. Selective action of the photosensitizer QLT0074 on activated human T lymphocytes. Photochem Photobiol. 2002; 76:224-231. [PubMed: 12194221]

23. Chen HN, Liu CM, Yang H, Chou HY, Chiang CP, Kuoet MYP. 5-Aminolevulinic acid induces apoptosis via NF- $\mathrm{\kappa B} / \mathrm{JNK}$ pathway in human oral cancer Ca9-22 cells. J Oral Pathol Med. 2011; 40:483-490. [PubMed: 21138480]

24. Wu RWK, Yow CMN, Wong CK, Lam YH. Photodynamic therapy (PDT) - initiation of apoptosis via activation of stress-activated p38 MAPK and JNK signal pathway in H460 cell lines. Photodiagnosis Photodyn Ther. 2011; 8:254-263. [PubMed: 21864799]

25. Ahmad N, Gupta S, Feyes DK, Mukhtar H. Involvement of Fas (APO-1/CD95) during photodynamic therapy-mediated apoptosis in human epidermoid carcinoma A431 cells. J Invest Dermatol. 2000; 115:1041-1046. [PubMed: 11121139]

26. Ali SM, Chee SK, Yuen GY, Olivo M. Photodynamic therapy induced Fas-mediated apoptosis in human carcinoma cells. Int J Mol Med. 2002; 9:257-270. [PubMed: 11836632]

27. Olivo M, Ali-Seyed M. Apoptosis-signalling mechnisms in human cancer cells induced by Calphostin-PDT. Int J Oncol. 2007; 30:537-548. [PubMed: 17273754]

28. Chen B, Roskams T, Xu Y, Agostinis P, De Witte PAM. Photodynamic therapy with hypericin induces vascular damage and apoptosis in the RIF-1 mouse tumor model. Int J Cancer. 2002; 98:284-290. [PubMed: 11857421]

29. Furre IE, Moller MTN, Shahzidi S, Nesland JM, Peng Q. Involvement of both caspase-dependent and -independent pathways in apoptotic induction by hexaminolevulinate-mediated photodynamic therapy in human lymphoma cells. Apoptosis. 2006; 11:2031-2042. [PubMed: 17036199]

30. Stutz N, Johnson RD, Wood GS. The Fas apoptotic pathway in cutaneous T-cell lymphomas: frequent expression of phenotypes associated with resistance to apoptosis. J Am Acad Dermatol. 2012; 67:1327. [PubMed: 22884443]

31. Kantari C, Walczak H. Caspase- 8 and bid: caught in the act between death receptors and mitochondria. Biochim Biophys Acta. 2011; 1813:558-563. [PubMed: 21295084]

32. Phillips DC, Woollard KJ, Griffiths HR. The anti-inflammatory actions of methotrexate are critically dependent upon the production of reactive oxygen species. Br J Pharmacol. 2003; 138:501-511. [PubMed: 12569075]

33. Sinha AK, Anand S, Ortel BJ, Chang Y, Mai Z, Hasan T, Maytin EV. Methotrexate used in combination with aminolevulinic acid for photodynamic killing of prostate cancer cells. Br J Cancer. 2006; 95:485-495. [PubMed: 16868543]

34. Anand S, Honari G, Hasan TP, Elson P, Maytin EV. Low-dose methotrexate enhances aminolevulinate-based photodynamic therapy in skin carcinoma cells in vitro and in vivo. Clin Cancer Res. 2009; 15:3333-3343. [PubMed: 19447864] 
35. Krathen M, Bashey S, Sutherland K, Sundram U, Nagpal S, Salva K, Wood G, Advani R, Hoppe RH, Reddy S, Pulitzer M, Horwitz S, Kim YH. Brentuximab Vedotin Demonstrates Significant Clinical Activity in Relapsed or Refractory Mycosis Fungoides. Blood. 2012; 120:797.

36. Wu J, Salva KA, Stutz N, Longley BJ, Spiegelman VS, Wood GS. Quantitative gene analysis of methylation and expression (Q-GAME) in fresh or fixed cells and tissues. Exp Dermatol. 2014; 23:304-9. [PubMed: 24646432]

37. Wu J, Salva KA, Wood GS. C-CBL E3 ubiquitin ligase is overexpressed in cutaneous T-cell lymphoma: its inhibition promotes activation-induced cell death. J Invest Dermatol. 2015; 135:861-868. [PubMed: 25140833]

38. DeSimone JA, Guenova E, Carter JB, Chaney KS, Aldridge JR, Noell CM, Dorosario AA, Hansen JL, Kupper TS, Devlin PM. Low-dose high-dose-rate brachytherapy in the treatment of facial lesions of cutaneous T-cell lymphoma. J Am Acad Dermatol. 2013; 69:61-65. [PubMed: 23453243]

39. Goddard AL, Vleugels RA, LeBoeuf NR, O'Farrell DA, Cormack RA, Hansen JL, Kupper TS, Devlin PM. Palliative therapy for recalcitrant cutaneous T-cell lymphoma of the hands and feet with low-dose, high-dose-rate brachytherapy. JAMA Dermatol. 2015; 151:1354-1357. [PubMed: 26397774]

40. Guix B, Finestres F, Tello J, Palma C, Martinez A, Guix J, Guix r. Treatment of skin carcinomas of the face by high-dose-rate brachytherapy and custom-made surface molds. Int J Radiat Oncol Biol Phys. 2000; 47:95-102. [PubMed: 10758310]

41. Garibyan L, Cotter SE, Hansen JL, Noell C, Dorosario A, O'Farrel DA, Devlin PM, Wang LC. Palliative treatment for in-transit cutaneous metastases of Merkel cell carcinoma using surface/ mold computer-optimized high-dose-rate brachytherapy. Cancer J. 2013; 19:283-287. [PubMed: 23867506] 


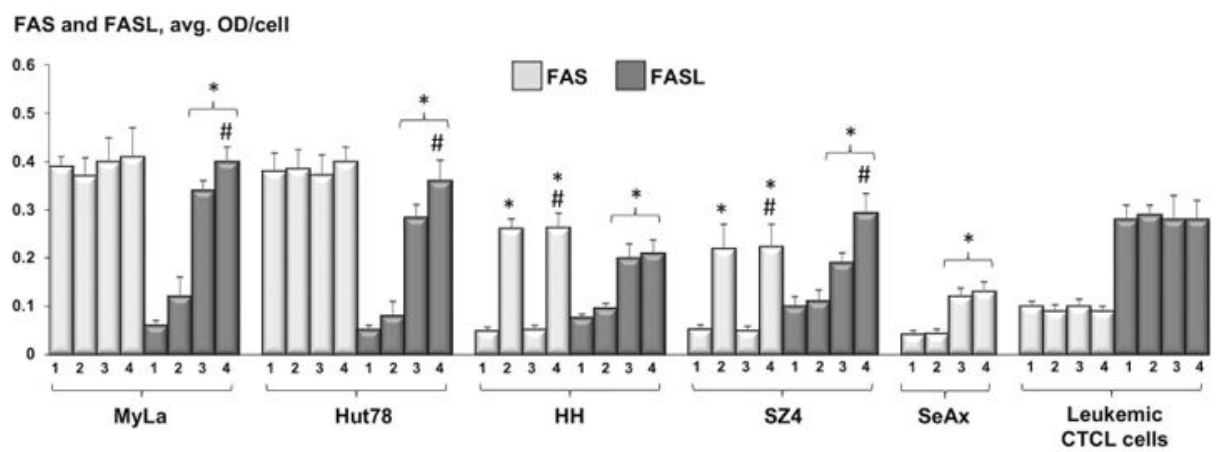

Figure 1.

Quantitative analysis of FAS and FASL-expression in CTCL cell lines and leukemic CTCL cells: untreated samples (1), post 48h incubation with $100 \mathrm{nM}$ MTX (2), 24 hours after standard PDT (3), and 24 hours following ePDT (4). * p $<0.05$ as compared to untreated samples; \# p $<0.05$ relative to standard PDT. 


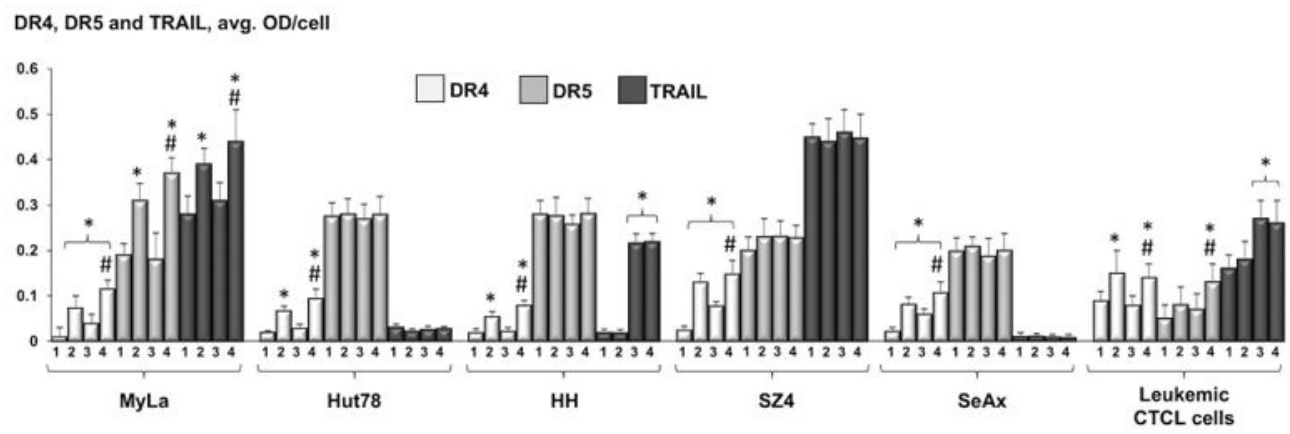

Figure 2.

Expression of DR4, DR5 and TRAIL in CTCL cell lines and leukemic CTCL cells as determined by MIA, showing untreated cells (1), cells post $48 \mathrm{~h}$ incubation with $100 \mathrm{nM}$ MTX (2), cells 24 hours after PDT alone (3), and cells 24 hours after ePDT (4). * p < 0.05 as compared to untreated samples; \# $\mathrm{p}<0.05$ relative to standard PDT. 


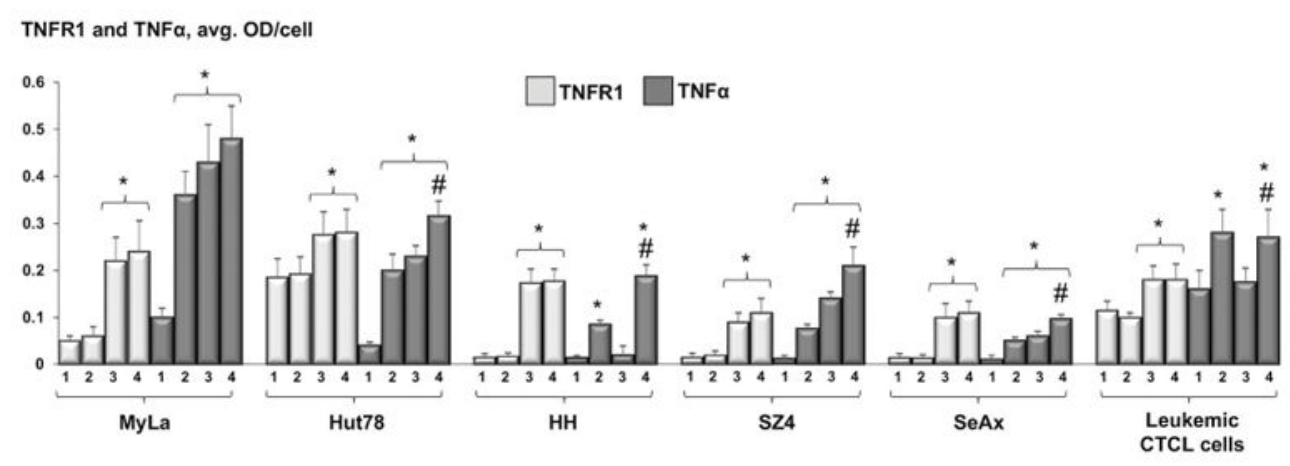

Figure 3.

MIA-quantitated expression of TNFR1 and TNFa in CTCL cell lines and leukemic CTCL cells: untreated samples (1), after 48h incubation with $100 \mathrm{nM}$ MTX (2), 24 hours after standard PDT (3), and 24 hours after ePDT (4). $*$ p $<0.05$ as compared to untreated samples; \# $\mathrm{p}<0.05$ relative to standard PDT. 


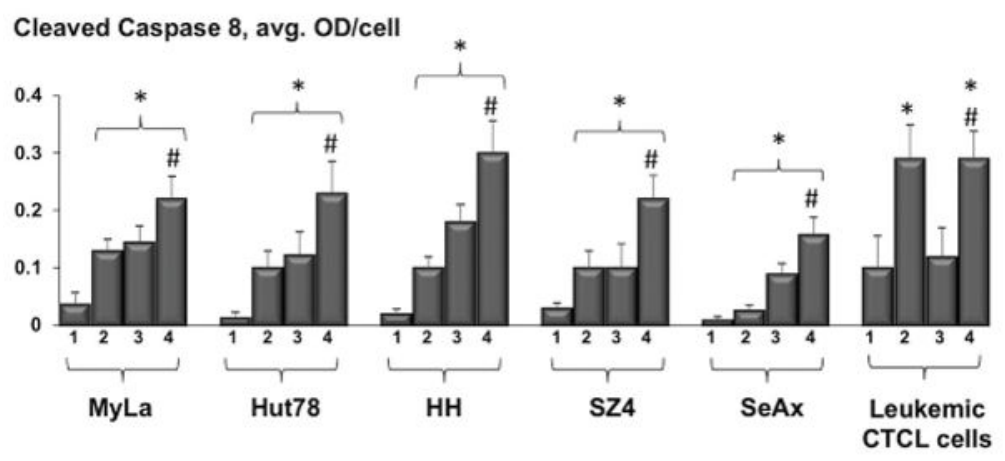

Figure 4.

Assessment of cleaved caspase 8 levels in CTCL cell lines and leukemic CTCL cells based on MIA: untreated samples (1), post MTX alone (2), post standard PDT (3) and post ePDT (4). ${ }^{*} \mathrm{p}<0.05$ as compared to untreated cells; $\# \mathrm{p}<0.05$ relative to standard PDT. 
Cleaved Caspase 3, avg. OD/cell

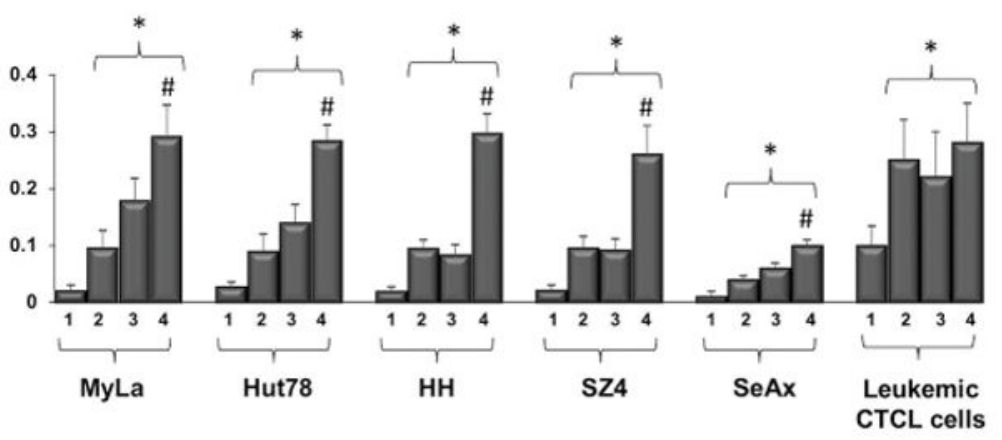

Figure 5.

MIA-assessed cleaved caspase 3 expression in CTCL cell lines and leukemic CTCL cells, showing untreated samples (1), cells post MTX alone (2), cells post standard PDT (3) and cells post ePDT (4). * $\mathrm{p}<0.05$ as compared to untreated samples; \# $\mathrm{p}<0.05$ relative to standard PDT. 
Cleaved Caspase 9, avg. OD/cell

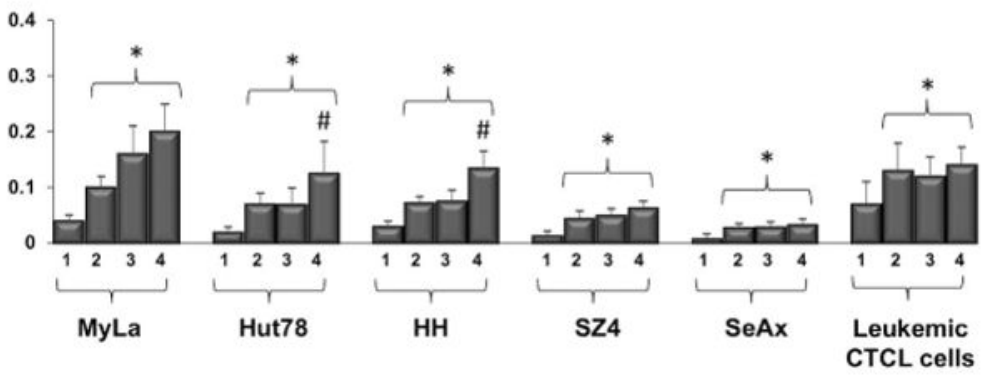

Figure 6.

MIA-determined levels of cleaved caspase 9 in CTCL cell lines and leukemic CTCL cells: untreated samples (1), post MTX alone (2), post PDT alone (3) and post ePDT (4). * p < 0.05 as compared to untreated samples; \# $\mathrm{p}<0.05$ relative to standard PDT. 


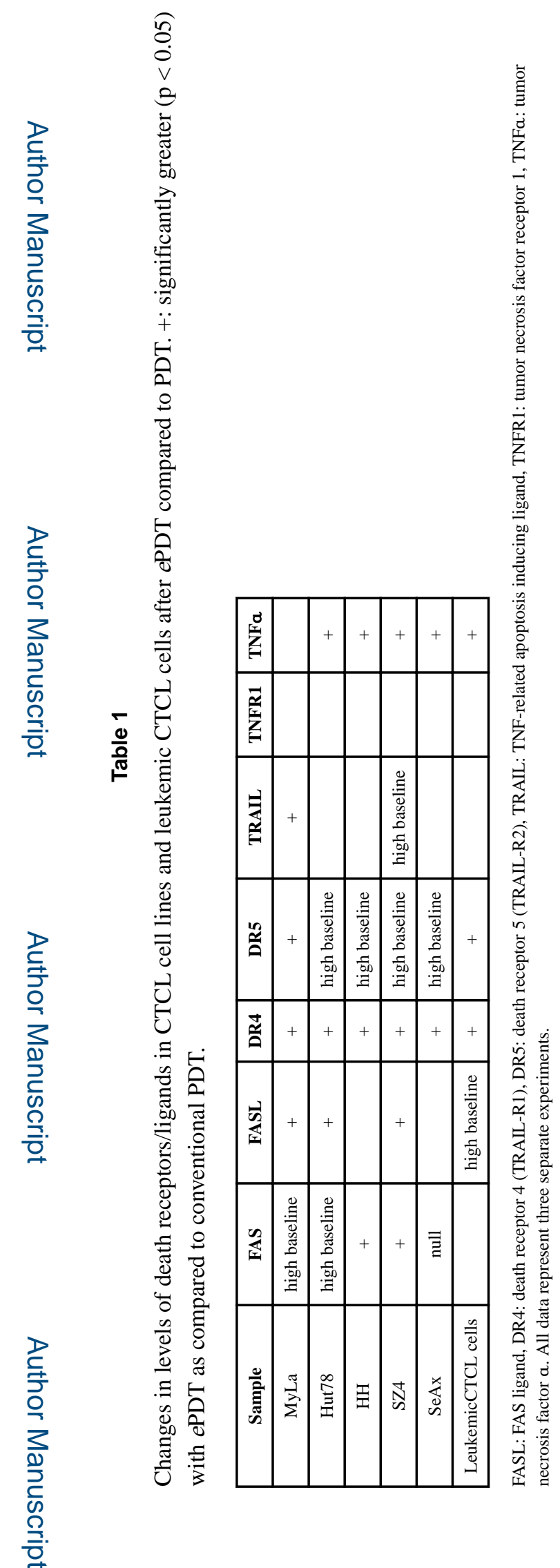

Photochem Photobiol. Author manuscript; available in PMC 2019 September 01. 


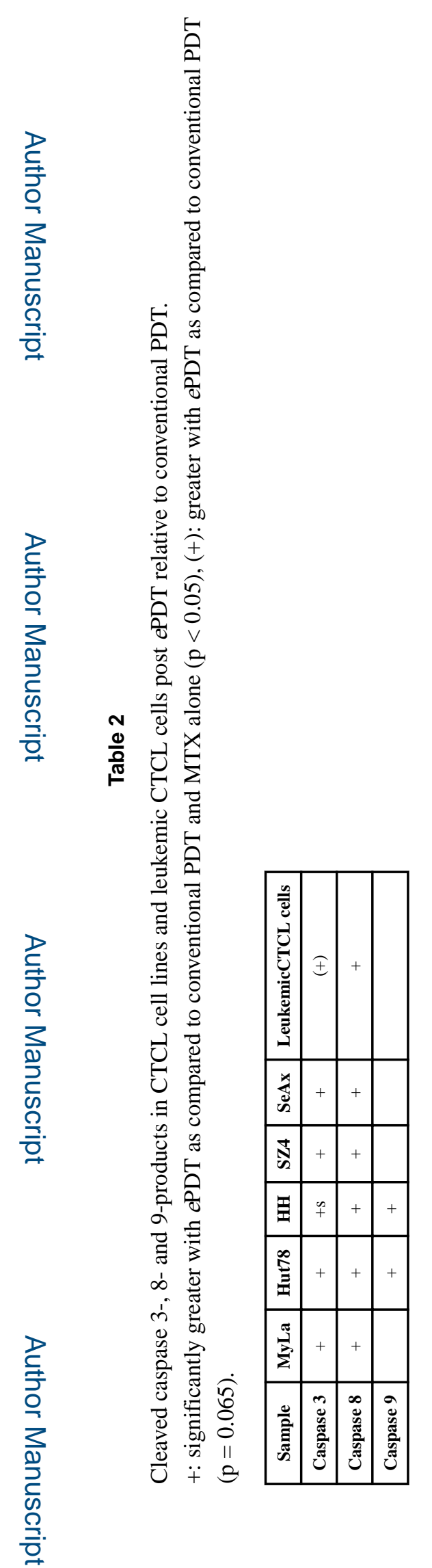

Photochem Photobiol. Author manuscript; available in PMC 2019 September 01. 